\title{
Association between sleep-related breathing disorders and academic performance among children from Concepción, Chile
}

\author{
Darwin Gatica, Kinesiologist ta,b, , Iván Rodríguez-Núñez, Kinesiologist ${ }^{d, h}$, Daniel Zenteno, M.D. ${ }^{e}$, \\ María J. Elso, M.D. $f$, Juan J. Montesinos, Kinesiologist ${ }^{e}$ and Carlos Manterola, M.D., ${ }^{c, g}$
}

\begin{abstract}
The objective of this study was to establish an association between academic performance in Math, Language Arts, and Science and the presence of sleep-related breathing disorders (SRBDs) among healthy schoolchildren from the city of Concepción, Chile. Healthy children were defined as those without comorbidities. Outcome measures of interest included the analysis of academic performance in Math, Language Arts, and Science and the presence of SRBD assessed using the Pediatric Sleep Questionnaire.

Two-hundred and fifty-six children were included in the study (59.8\% were boys). In the studied sample, SRBD prevalence was $24.6 \%$. A significant association was observed between SRBD and a low performance in Math (odds ratio [OR]: 3.1, 1.5-6.8), Language Arts (OR:2.5, 1.1-5.5), and Science (OR: 4.2, 1.7-10.0). To conclude, in the studied sample, the presence of SRBD was associated with a low academic performance in Language Arts, Math, and Science.

Key words: obstructive sleep apnea, child, snoring, neurocognitive disorder, surveys and questionnaires.
\end{abstract}

http:/ / dx.doi.org/10.5546/ aap.2017.eng.497

To cite: Gatica D, Rodríguez-Núñez I, Zenteno D, et al. Association between sleep-related breathing disorders and academic performance among children from Concepción, Chile. Arch Argent Pediatr2017;115(5):497-500.

a. School of Kinesiology, School of Health, Universidad Santo Tomás, Concepción, Chile.

b. Sleep Center, ClínicaUniversitaria de San Pedro de la Paz, Chile.

c. Doctoral Program in Medical Sciences, School of Medicine, Universidad de La Frontera, Temuco, Chile.

d. Exercise Biology Lab, School of Kinesiology, School of Health Sciences, Universidad San Sebastián, Concepción, Chile.

e. Department of Pediatrics, Universidad de Concepción. Division of Pediatrics, Hospital Guillermo Grant Benavente, Concepción, Chile.

f. Department of Medical Specialties, Universidad de Concepción. Division of Neurology, Hospital Guillermo Grant Benavente, Concepción, Chile.

g. BiomedicalSciencesResearch Center, Universidad Autónoma de Chile.

h. Research Task Force in Cardiovascular and Respiratory Health IDEAS-CVR, Concepción, Chile.

E-mail addres:

Iván Rodríguez Núñez, Kinesiologist: ivan.rodriguez@uss.cl

Funding: None.

Conflict of interest: None.

Received: 11-21-2016

Accepted: 4-20-2017

\section{INTRODUCTION}

Sleep-related breathing disorders (SRBDs) describe a group of conditions, from simple snoring (primary snoring) to obstructive sleep apnea (OSA). ${ }^{1}$ OSA is characterized by intermittent, partial or complete upper airway obstruction, ${ }^{2}$ with a worldwide prevalence of up to $4 \%$ among children, and it is the cause of up to $18 \%$ of habitual snoring among Chilean children and adolescents. ${ }^{3,4}$ Considering that the standard method for SRBD diagnosis is a polysomnography, which results in a high economic burden on the health system, indirect methods have been developed to detect OSA. One of these indirect methods is the Pediatric Sleep Questionnaire (PSQ), developed by Chervin et al. ${ }^{5}$ The PSQ has been validated into Spanish by Vila et al., ${ }^{6}$ and used in Chile as is, without a crosscultural adaptation, although it offers a sensitivity of 0.714 , a specificity of 0.521 , a positive predictive value of 0.521 , and a negative predictive value of $0.714 .^{7}$

SRBD has been related to the development of cardiovascular and metabolic diseases in the pediatric population. ${ }^{8,9} \mathrm{In}$ addition, there is evidence of an association between SRBD symptoms and neurocognitive disorders, which suggests that SRBD may be a potential cause of low academic performance in the student population. ${ }^{10,12}$ However, in our setting, studies aimed at establishing an association between the risk for SRBD and academic performance are scarce.

The objective of this study was to establish an association between performance in Math, Language Arts, and Science and SRBD using the PSQ among schoolchildren from the city of Concepción, Chile.

\section{MATERIAL AND METHOD}

Design: Cross-sectional study.

Participants: Among 60 schools, schoolchildren aged 6-14 years were selected by convenience sampling from 4 schools: 1 public school, 2 private schools, and 1 state-subsidized institution from the city of Concepción, Chile. Male and female children whose parents agreed to 
TABLE 1. Biodemographic characteristics, results of the pediatric sleep questionnaire and overall academic performance of the 256 children included in the sample

\begin{tabular}{cc}
\hline $\begin{array}{c}\text { Biodemographic } \\
\text { characteristics }\end{array}$ & $\begin{array}{c}\text { Average } \pm \text { SD/ratio } \\
(\%)\end{array}$ \\
\hline Age (years old) & $9.23 \pm 2.3$ \\
Sex $(\mathrm{M} / \mathrm{F})$ & $153(59.8 \%) / 103(40.2 \%)$ \\
Weight $(\mathrm{kg})$ & $37.4 \pm 12.7$ \\
Height $(\mathrm{m})$ & $1.4 \pm 0.2$ \\
Asthma & $24(9.4 \%)$ \\
Rhinitis & $34(13.3 \%)$ \\
Attention deficit hyperactivity disorder & $6(2.3 \%)$ \\
PSQ (score) & $0.22 \pm 0.19$ \\
Risk for SRBD & $63(24.6 \%)$ \\
Years of education & \\
1 & $45(17.6 \%)$ \\
2 & $49(19.1 \%)$ \\
3 & $43(16.8 \%)$ \\
4 & $22(8.6 \%)$ \\
5 & $21(8.2 \%)$ \\
6 & $33(12.9 \%)$ \\
7 & $12(4.7 \%)$ \\
8 & $31(12.1 \%)$ \\
Language Arts average grade & $6.3 \pm 2.7$ \\
Science average grade & $5.8 \pm 0.9$ \\
Math average grade & $5.7 \pm 0.9$ \\
\hline
\end{tabular}

SD: standard deviation; M: male; F: female; PSQ: Pediatric Sleep Questionnaire; SRBD: sleep-related breathing disorder. Age, weight, height, PSQ, and average grades are shown as average \pm SD.

Sex, comorbidities, risk for SRBD, and years of education are shown as absolute value and percentage. participate in the study were included. Children with chronic diseases reported by their parents, such as chronic cardiovascular, respiratory and/

TABLE 2. Partial correlations between academic performance and the Pediatric Sleep Questionnaire score

\begin{tabular}{lcc}
\hline & PSQ (score) & $\boldsymbol{P}$ value \\
\hline Math & -0.35 & $<0.0001$ \\
Language Arts & -0.36 & $<0.0001$ \\
Science & -0.34 & $<0.0001$ \\
General average & -0.02 & 0.638 \\
\hline
\end{tabular}

PSQ: Pediatric Sleep Questionnaire.

Partial correlations were adjusted by years of education and comorbidities.

TABLE 3. Logistic regression analysis based on the association between the risk for sleep-related breathing disorders and low academic performance

\begin{tabular}{lcc}
\hline & OR (95\% CI) & $\boldsymbol{P}$ value \\
\hline Low performance in Math & $3.1(1.5-6.8)$ & 0.003 \\
Low performance in Language Arts & $2.5(1.1-5.5)$ & 0.023 \\
Low performance in Science & $4.2(1.7-10.0)$ & 0.002 \\
\hline
\end{tabular}

OR: odds ratio; CI: confidence interval.

Results are described as absolute value and 95\% CI.

ORs correspond to the Mantel-Haenszel adjusted OR by years of education and comorbidities.

FIgURE 1. Academic performance in Math, Language Arts, and Science among children at risk for sleep-related breathing disorder (N: 63) and without a risk for sleep-disordered breathing (N: 193)

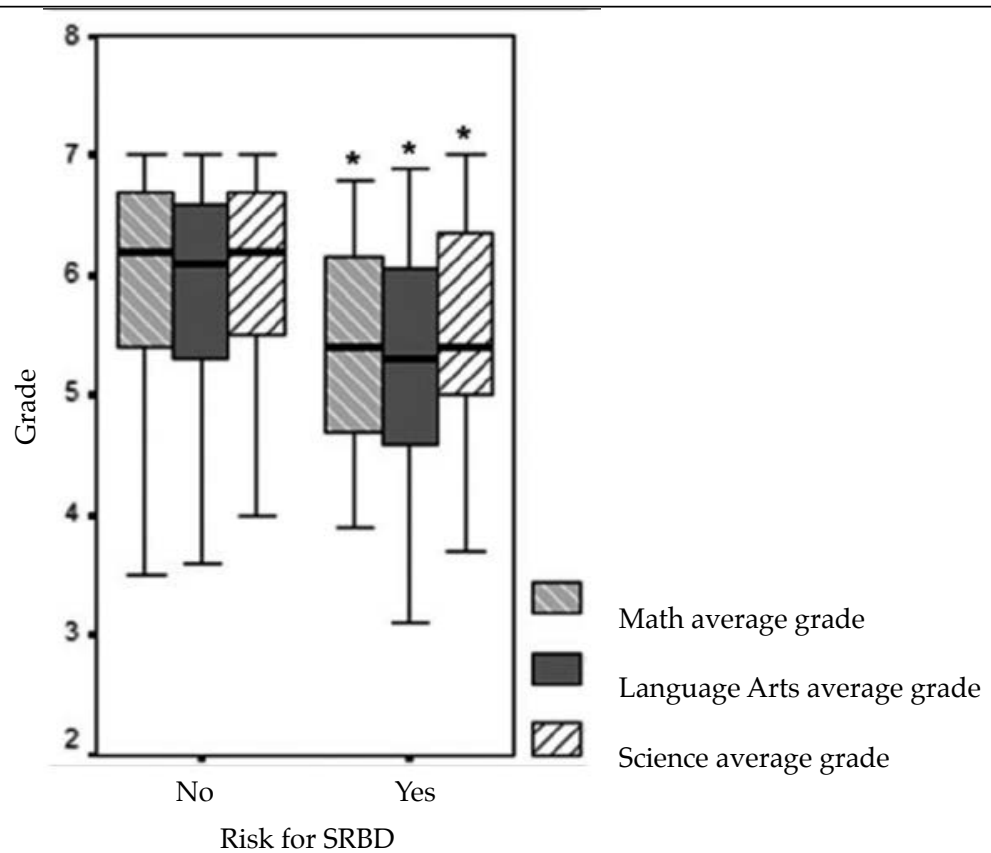

${ }^{*} \mathrm{p}<0.0001$; SRBD: sleep-related breathing disorder. 
or neuromuscular disease, cognitive disorders, and acute conditions in the past month were excluded. Children with incomplete data in the PSQ were also excluded. The study was conducted between March and August 2012.

Outcome measures: Each parent or caregiver completed a questionnaire with their child's age, sex, weight, height, and comorbidities. Body mass index (BMI) was estimated based on the weight/ (height) ${ }^{2}$ formula and described as $\mathrm{Z}$ score for age based on the World Health Organization's reference values.

Academic performance and the presence of SRBD were the outcome measures of interest. Academic performance was considered as the average grade from 1 to 7 in Math, Science, and Language Arts. Grades were obtained directly from academic records by a blinded investigator. In addition, symptoms related to SRBD were assessed using the abridged version of the PSQ (see Annex). Before starting the study, the understanding of questionnaire items was assessed in a sample of individuals that matched the parents or caregivers of the studied children.

The questionnaire was completed by the children's parents or caregivers at home. Parents received the questionnaire together with basic instructions on how to complete it. ${ }^{6}$ Finally, completed questionnaires were returned to the teachers of each class, who sent them directly to investigators.

The PSQ covers 3 domains: the first domain includes 8 items related to the child's behavior during the night and while sleeping; the second one has 7 items regarding the child's behavior during the day and other potential problems, such as delayed growth and obesity; and the third one includes questions related to the attention deficit hyperactivity disorder from the Diagnostic and Statistical Manual of Mental Disorders, fourth edition (DSM-IV). For the first and second domains, the response format was yes/no/don't know. For the third domain, answer categories were never/sometimes/often/almost always. However, for analysis purposes, often/almost always were categorized as "yes" whereas never/ sometimes were categorized as "no." To estimate the final score, the sum of all yes answers was divided into the total number of yes / no answers. Thus, a score above 0.33 indicated the presence of SRBD in accordance with what was published by Bertrán et al. ${ }^{6}$

Statistical analysis: Normality was measured using the Kolmogorov-Smirnov test. Descriptive statistics were established subsequently using average and standard deviation for quantitative outcome measures and percentages, for qualitative outcome measures.

To establish the association between the PSQ score and academic performance, a partially-adjusted correlation coefficient was estimated based on years of education, BMI, and comorbidities. In addition, Student's t test was used for independent samples to compare academic performance between children with and without SRBD.

Finally, a logistic regression analysis was done using the odds ratio (OR) computed with the Mantel-Haenszel method, adjusted by years of education and comorbidities; homogeneity of the OR was estimated using the Breslow-Day test. ORs were described as absolute value and $95 \%$ confidence interval (CI). A $p$ value of $<0.05$ was considered significant.

Ethical principles: Every child signed an informed assent, and parents signed an informed consent to authorize their children's participation in the study. The study was approved by the Institutional Ethics Committee.

\section{RESULTS}

Two-hundred and fifty-six children were included in the study (59.8\% were boys), which accounted for a response rate of $51.4 \%$. Their mean weight and height were $37.4 \pm 12.7 \mathrm{~kg}$ and $1.4 \pm 0.2 \mathrm{~m}$. Their BMI Z score was $1.1 \pm 1.2 \mathrm{~kg} / \mathrm{m}^{2}$ (interquartile range: 0.35-1.86). A low percentage of the sample had some comorbidities; the most common comorbidity was rhinitis $(13.3 \%)$, followed by asthma (9.4\%), and attention deficit hyperactivity disorder $(2.3 \%)$. The average PSQ score was $0.22 \pm 0.19$ and the prevalence of children with SRBD was $24.6 \%$ (95\% CI: 30.6-18.6). Biodemographic characteristics and academic history of the sample population are detailed in Table 1.

Children at risk for SRBD had a lower academic performance compared to children without risk for SRBD (Figure 1). In addition, the PSQ score showed a significantly negative correlation with academic performance in Math, Language Arts, and Science (Table 2).

The logistic regression analysis showed an association between the PSQ score and academic performance in the studied areas (Table 3).

Lastly, no significant difference was observed in the size of the OR between public and private schools. 


\section{DISCUSSION}

The results of this study indicated that the presence of SRBD, as assessed using the PSQ, had a negative impact on the neurocognitive ability of schoolchildren, which is evidenced in their academic performance inLanguage Arts, Math, and Science.

Our findings are consistent with those observed in previous studies. In this context, Galland et al. have recently demonstrated, using a meta-analysis approach, that SRBD was associated with poor academic performance in Language Arts, Math, and Science and learning difficulties. ${ }^{13}$ In addition, in Chile, Brockmann et al. observed a strong association between SRBD and a low academic performance in Language Arts and Physical Education; however, it was not possible to determine such association with Math and Science. ${ }^{4}$

In the study sample, SRBD prevalence was $24.6 \%$, which was higher than what had been observed in previous studies conducted in other Chilean cities (18\%). ${ }^{4}$ This suggests that the association between SRBD and academic performance may be independent from SRBD prevalence in the study population. ${ }^{4}$

Some studies have shown a higher SRBD prevalence in schools located in lower socioeconomic settings. ${ }^{6,12-14}$ Notwithstanding this, our findings did not show significant differences in SRBD prevalence or in the association between SRBD and academic performance between public and private schools.

More recently, an association has been observed between SRBD severity and neurocognitive disorders, which suggests that having a SRBD has an impact on children's neurocognitive ability by means of a doseresponse relationship. ${ }^{15}$

In our study, the abridged version of the PSQ was used, which assessed SRBD-related symptoms only; however, it was not possible to establish their severity. ${ }^{5}$ Therefore, in our findings it is not possible to establish such association, which should be addressed in future research.

A weakness of this study is that the determination of sample exclusion criteria was the responsibility of parents, which makes it impossible to rule out a selection bias. In addition, although the PSQ has been used in Chile before, it has not been cross-culturally adapted to the Chilean population, which may result in a potential measurement bias.

Finally, it is possible to conclude that, in the studied sample, the presence of SRBD was associated with a low academic performance in Language Arts, Math, and Science. Future studies are required to establish the relationship between severity and effect.

\section{REFERENCES}

1. Zenteno D, Salinas P, Vera R, et al. Enfoque Pediátrico para el Estudio de los Trastornos Respiratorios del Sueño. Rev Chil Pediatr 2010;81(5):445-55.

2. Elso MJ, Brockmann P, Zenteno D. Consecuencias del síndrome de apnea obstructiva del sueño. Rev Chil Pediatr 2013;84(2):128-37.

3. Lumeng JC, Chervin RD. Epidemiology of pediatric obstructive sleep apnea. Proc Am Thorac Soc 2008;5(2): 242-52.

4. Brockmann PE, Bertrand P, Pardo T, et al. Prevalence of habitual snoring and associated neurocognitive consequences among Chilean school aged children. Int $J$ Pediatr Otorhinolaryngol 2012;76(9):1327-31.

5. Chervin RD, Hedger K, Dillon JE, et al. Pediatric sleep questionnaire (PSQ): validity and reliability of scales for sleep-disordered breathing, snoring, sleepiness, and behavioral problems. Sleep Med 2000;1(1):21-32.

6. Tomás Vila M, Miralles Torres A, Beseler Soto B. Versión española del PediatricSleepQuestionnaire. Uninstrumento útil en la investigación de los trastornos del sueño en la infancia. Análisis de sufiabilidad. An Pediatr (Barc) 2007;66(2):121-8.

7. Bertran K, Mesa T, Rosso K, et al. Diagnostic accuracy of the Spanish version of the Pediatric Sleep Questionnaire for screening of obstructive sleep apnea in habitually snoring children. Sleep Med 2015;16(5):631-6.

8. GozalD.Sleep, sleep disorders and inflammation in children. Sleep Med 2009;10(Suppl 1):S12-6.

9. Quist JS, Sjödin A, Chaput JP, et al. Sleep and cardiometabolic risk in children and adolescents. Sleep Med Rev 2016; 29:76-100.

10. De Carvalho LB, Do Prado LB, Ferreira V, et al. Symptoms of sleep disorders and objective academic performance. Sleep Med 2013;14(9):872-6.

11. Gozal D. Sleep-disordered breathing and school performance in children. Pediatrics 1998;102(3 Pt 1):616-20.

12. Chervin RD, ClarkeD, Huffman J, etal.School performance, race, and other correlates of sleep-disordered breathing in children. Sleep Med 2003;4(1):21-7.

13. Galland B, Spruyt K, Dawes P, et al. Sleep disordered breathing and academic performance: a meta-analysis. Pediatrics 2015;136(4):e934-46.

14. Friberg D, Lundkvist K, Li X, et al. Parental poverty and occupation as risk factors for pediatric sleep-disordered breathing. Sleep Med 2015;16(9):1169-75.

15. HunterSJ, GozalD, SmithDL, etal.Effect of sleep-disordered breathing severity on cognitive performance measures in a large community cohort of young school-aged children. Am J Respir Crit Care Med 2016;194(6):739-47. 
ANNEX

Abridged version of the Pediatric Sleep Questionnaire

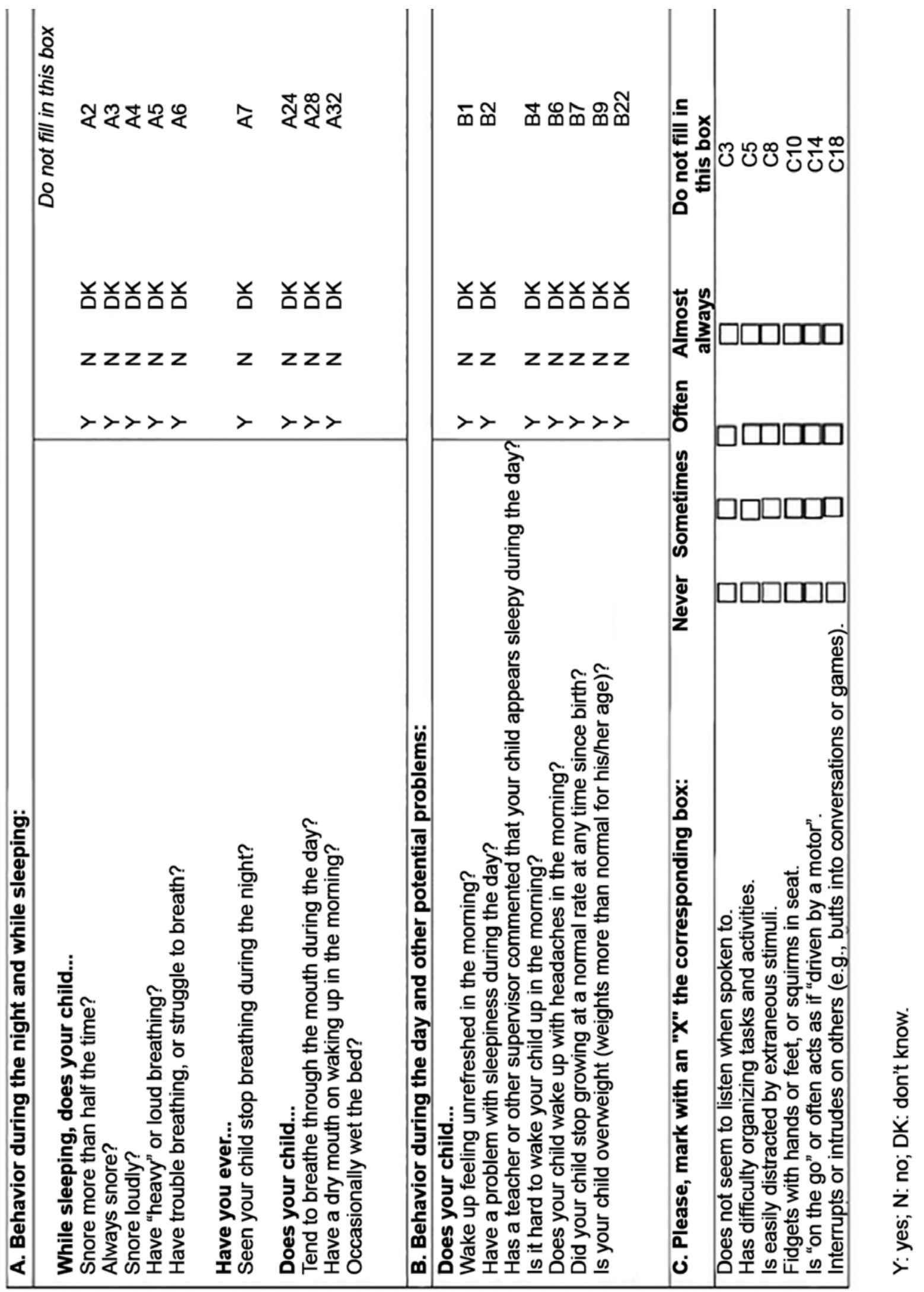

